\title{
THURSTON MAPS AND ASYMPTOTIC UPPER CURVATURE
}

\author{
QIAN YIN
}

\begin{abstract}
A Thurston map is a branched covering map from $\mathbb{S}^{2}$ to $\mathbb{S}^{2}$ with a finite postcritical set. We associate a natural Gromov hyperbolic graph $\mathcal{G}=\mathcal{G}(f, \mathcal{C})$ with an expanding Thurston map $f$ and a Jordan curve $\mathcal{C}$ on $\mathbb{S}^{2}$ containing post $(f)$. The boundary at infinity of $\mathcal{G}$ with associated visual metrics can be identified with $\mathbb{S}^{2}$ equipped with the visual metric induced by the expanding Thurston map $f$. We define asymptotic upper curvature of an expanding Thurston map $f$ to be the asymptotic upper curvature of the associated Gromov hyperbolic graph, and establish a connection between the asymptotic upper curvature of $f$ and the entropy of $f$.
\end{abstract}

\section{Contents}

1. Introduction

2. Expanding Thurston maps and Cell Decompositions

3. Gromov Hyperbolic Spaces

4. Tile Graphs

5. Asymptotic Upper Curvature

References

\section{INTRODUCTION}

A rational map $f: \widehat{\mathbb{C}} \rightarrow \widehat{\mathbb{C}}$ is a map on the Riemann sphere $\widehat{\mathbb{C}}=\mathbb{C} \cup\{\infty\}$ which can be written as a quotient of two relatively prime complex polynomials $p(z)$ and $q(z)$, with $q(z) \neq 0$,

$$
f(z)=\frac{p(z)}{q(z)}=\frac{a_{0} z^{m}+\ldots+a_{m}}{b_{0} z^{l}+\ldots+b_{l}}
$$

where $a_{i}, b_{j} \in \mathbb{C}$ for $i=0, \ldots, m$ and $j=0, \ldots, l$. The postcritical set post $(f)$ of $f$ is defined to be the forward orbits of the critical points

$$
\operatorname{post}(f)=\bigcup_{n \geq 1}\left\{f^{n}(c): c \in \operatorname{crit}(f)\right\} .
$$

The author was partially supported by NSF grants DMS 0757732, DMS 0353549, DMS 0456940, DMS 0652915, DMS 1058772, and DMS 1058283. 
If the postcritical set post $(f)$ is finite, we say that the map $f$ is postcritically finite.

Thurston introduced a topological analog of a postcritically finite rational map, now known as a Thurston map (see [DH]). A Thurston map $f: \mathbb{S}^{2} \rightarrow \mathbb{S}^{2}$ is a branched covering map with finite postcritical set post $(f)$. The notion of an expanding Thurston map was introduced in [BM] as a topological analog of a postcritically finite rational map whose Julia set is the whole sphere $\widehat{\mathbb{C}}$. Roughly speaking, a Thurston map is called expanding if all the connected components of the preimage under $f^{-n}$ of any open Jordan region disjoint from post $(f)$ become uniformly small as $n$ tends to infinity. We refer the reader to Definition 2.1 for a more precise statement. A related and more general notion of expanding Thurston maps was introduced in [HP]. Lattès maps are among the simplest examples of expanding Thurston maps.

Let $f$ be an expanding Thurston map, and let $\mathcal{C}$ be a Jordan curve containing post $(f)$. The Jordan Curve Theorem implies that $\mathbb{S}^{2} \backslash \mathcal{C}$ has precisely two connected components, whose closures we call 0-tiles. We call the closure of each connected component of the preimage of $\mathbb{S}^{2} \backslash \mathcal{C}$ under $f^{n}$ an $n$-tile. In Section 5 of [BM], it is proved that the collection of all $n$-tiles gives a cell decomposition of $\mathbb{S}^{2}$.

Every expanding Thurston map $f: \mathbb{S}^{2} \rightarrow \mathbb{S}^{2}$ induces a natural class of metrics on $\mathbb{S}^{2}$, called visual metrics (see Definition 2.11), and each visual metric $d$ has an associated expansion factor $\Lambda>1$. This visual metric is essentially characterized by the geometric property that the diameter of an $n$-tile is about $\Lambda^{-n}$, and the distance between two disjoint $n$-tiles is at least about $\Lambda^{-n}$. The supremum of the expansion factors of all visual metrics is called the combinatorial expansion factor $\Lambda_{0}$ (see [BM, Theorem 1.5]). For Lattès maps, the supremum is obtained. In general, the supremum is not obtained.

A geodesic metric space $(X, d)$ is called a Gromov hyperbolic space if every geodesic triangle in it is "very thin". It can also defined in terms of Gromov products. For any points $x, y, p \in X$, the Gromov product $(x, y)_{p}$ of $x$ and $y$ with respect to the base point $p$ is defined as

$$
(x, y)_{p}:=\frac{1}{2}[d(x, p)+d(y, p)-d(x, y)] .
$$

The space $X$ is called $\delta$-hyperbolic (or Gromov hyperbolic) for some $\delta \geq 0$ if there exists a base point $p \in X$ such that for all $x, y, z \in X$, we have

$$
(x, y)_{p} \geq \min \left\{(x, z)_{p},(z, y)_{p}\right\}-\delta .
$$

We construct a graph $\mathcal{G}=\mathcal{G}(f, \mathcal{C})$ by letting the tiles in the cell decompositions of $(f, \mathcal{C})$ be vertices of $\mathcal{G}$. There is an edge between the two vertices $X^{n}, Y^{m} \in V$, denoted $X^{n} \sim Y^{m}$ if as underlying tiles

$$
|n-m| \leq 1 \text { and } X^{n} \cap Y^{m} \neq \emptyset \text {. }
$$


It turns out that the graph $\mathcal{G}$ with the path metric is a Gromov hyperbolic space (see Theorem 4.5).

Theorem 1.1. Let $f: \mathbb{S}^{2} \rightarrow \mathbb{S}^{2}$ be an expanding Thurston map and let $\mathcal{C} \subset \mathbb{S}^{2}$ be a Jordan curve containing post $(f)$. Then the graph $\mathcal{G}(f, \mathcal{C})$ equipped with the path metric $\eta$ is a Gromov hyperbolic space.

There is a natural boundary at infinity of a Gromov hyperbolic space. Roughly speaking, the boundary at infinity is the set of equivalence classes of geodesic rays in the Gromov hyperbolic space. It can also be equipped with a Gromov product by taking infimum of the infimum limit of the Gromov product along all the geodesic rays among the corresponding equivalence classes. A visual metric $\rho$ on the boundary at infinity of a Gromov hyperbolic space is a metric that has a bounded ratio

$$
\rho\left(\xi, \xi^{\prime}\right) / \Lambda^{-\left(\xi, \xi^{\prime}\right)_{p}}
$$

for some fixed $\Lambda>1$ and for all points $\xi$ and $\xi^{\prime}$ on the boundary.

In Proposition 4.7, we show the following:

Proposition 1.2. For an expanding Thurston map $f$ and a Jordan curve $\mathcal{C} \subset \mathbb{S}^{2}$ containing post $(f)$, the boundary at infinity $\partial_{\infty} \mathcal{G}$ of the graph tile $\mathcal{G}(f, \mathcal{C})$ can be identified with $\mathbb{S}^{2}$. Under this identification, a metric $d$ is a visual metric on $\mathbb{S}^{2}$ with respect to the expanding Thurston map $f$ if and only if $d$ is a visual metric on $\partial_{\infty} \mathcal{G}$ (in the sense of Gromov hyperbolic spaces).

We deduce that for any Jordan curves $\mathcal{C}$ and $\mathcal{C}^{\prime}$ containing post $(f)$, the classes of visual metrics on $\partial_{\infty} \mathcal{G}(f, \mathcal{C})$ and $\partial_{\infty} \mathcal{G}\left(f, \mathcal{C}^{\prime}\right)$ can also be identified (see Corollary 4.8). A similar graph to $\mathcal{G}(f, \mathcal{C})$ has also been studied by Kevin Pilgrim in $[\mathrm{P}$, from a somewhat different point of view. Our results overlap in some special cases. He considers the map $f$ being $C^{1}$ and $\mathbb{S}^{2} \backslash$ post $(f)$ equipped with a special Riemannian metric, and prove that the Julia set of $f$ can be identified as the Gromov boundary of a certain Gromov hyperbolic one-complex.

In $[\mathrm{BF}]$, the asymptotic upper curvature of a Gromov hyperbolic space is introduced. It is the analog of sectional curvature on Riemannian manifolds. Fix $\kappa \in[-\infty, 0)$. We call a metric space $X$ an $A C_{u}(\kappa)$-space if there exists $p \in X$ and a constant $c \geq 0$ such that for all $x, x^{\prime} \in X$ and all finite sequences $x_{0}=x, x_{1}, \ldots, x_{n}=x^{\prime}$ in $X$,

$$
\left(x, x^{\prime}\right)_{p} \geq \min _{i=1, \ldots, n}\left(x_{i-1}, x_{i}\right)_{p}-\frac{1}{\sqrt{-\kappa}} \log n-c .
$$

Here we use the convention $1 / \sqrt{\infty}=0$. We call

$$
K_{u}(X):=\inf \left\{\kappa \in[-\infty, 0): X \text { is an } \mathrm{AC}_{u}(\kappa) \text {-space }\right\}
$$

the asymptotic upper curvature of $X$. It is invariant under roughisometry. 
For any Jordan curves $\mathcal{C}$ and $\mathcal{C}^{\prime}$ containing post $(f)$, the metric spaces $\mathcal{G}=\mathcal{G}(f, \mathcal{C})$ and $\mathcal{G}^{\prime}=\mathcal{G}\left(f, \mathcal{C}^{\prime}\right)$ are rough-isometric (see Proposition 4.6). Hence we may define the asymptotic upper curvature $K_{u}(f)$ of an expanding Thurston map $f$ as

$$
K_{u}(f):=K_{u}(\mathcal{G}(f, \mathcal{C})),
$$

where $\mathcal{C} \subseteq \mathbb{S}^{2}$ is any Jordan curve containing post $(f)$. Using the notation above, we have the following theorem (see Theorem [5.3).

Theorem 1.3. Let $f: \mathbb{S}^{2} \rightarrow \mathbb{S}^{2}$ be an expanding Thurston map. The asymptotic upper curvature of $f$ satisfies

$$
K_{u}(f) \geq-\frac{1}{4} \log ^{2}(\operatorname{deg} f) .
$$

If in addition, the map $f$ has no periodic critical points, then the tile graph $\mathcal{G}=\mathcal{G}(f)$ is an $A C_{u}(\kappa)$-space with

$$
\kappa=-\frac{1}{4} \log ^{2}(\operatorname{deg} f),
$$

if and only if the map $f$ is topologically conjugate to a Lattès map.

Finally, we explain how Theorem 1.3 may be interpreted as adding a new and important piece to the Sullivan dictionary. The Sullivan dictionary is a collection of correspondences between concepts and results from the field of Kleinian groups and the field of iterated maps on $\mathbb{S}^{2}$.

The dictionary first appeared alongside the proof of the no wandering domain theorem [S]. Recall that the Fatou set of a rational map $f$ on the Riemann sphere is the set of points whose nearby points stay close together under iteration of $f$. The no wandering domain theorem states that each connected component of the Fatou set is eventually periodic under iteration by $f$. Sullivan proved this result, observing that it was analogous to Ahlfors' finiteness theorem [A]. Recall that a Kleinian group $\Gamma$ acts on the Riemann sphere via Möbius transformations, and that its domain of discontinuity is the largest open subset of the Riemann sphere on which $\Gamma$ acts properly discontinuously. If $\Gamma$ is finitely generated, then Ahlfors' finiteness theorem implies that every connected component of the domain of discontinuity has a non-trivial stabilizer, i.e. there are no wandering domains.

Sullivan argued that the Fatou set of a rational map plays an analogous role to the domain of discontinuity of a Kleinian group, along with many other such correspondences (see Table 1 for a sample, as well as CRSR, Section 2]). Since its original appearance in [S], the Sullivan dictionary has been significantly developed, and has continued to provide inspiration for research across both fields. For example, the recently proved density theorem for Kleinian groups [NS, O] provides a counterpart to the classical density theorem for structurally stable rational maps [MSS]. 
THURSTON MAPS AND ASYMPTOTIC UPPER CURVATURE

\begin{tabular}{|c|c|}
\hline Dynamical Systems & Kleinian Group \\
\hline Fatou sets & Domains of discontinuity \\
\hline Julia sets & Limit sets \\
\hline Periodic points & Fixed points \\
in the Julia set & $\begin{array}{c}\text { Loxodromic fixed points are dense } \\
\text { in the limit set }\end{array}$ \\
\hline $\begin{array}{c}\text { Mandelbrot set } \\
\text { Parameter space }\end{array}$ \\
\hline $\begin{array}{c}\text { Sullivan's no wandering domain theorem } \\
\text { of Fatou cycles }\end{array}$ & Ahlfors' finiteness theorem \\
\hline $\begin{array}{c}\text { Shishikura's sharp bound on number } \\
\text { Density theorem of structural stable maps }\end{array}$ & Density theorem for Kleinian groups \\
\hline $\begin{array}{c}\text { No invariant line fields on Julia set } \\
\text { (conjecture) }\end{array}$ & $\begin{array}{c}\text { No invariant line fields on limit set } \\
\text { Theorem 1.3 }\end{array}$ \\
\hline
\end{tabular}

TABLE 1. Sullivan dictionary

One famous result from the field of Kleinian groups with no known analogue under the Sullivan dictionary is Hamenstädt's entropy rigidity theorem $[\mathrm{H}]$, which establishes a connection between the curvature of a compact manifold $M$ and the topological entropy of the geodesic flow on the tangent bundle of $M$. More precisely, let $h(N)$ denote the topological entropy of the geodesic flow on the unit tangent bundle of a Riemannian manifold $N$. The theorem states that if $M$ is homotopic to a compact quotient $S$ of a hyperbolic space and $h(M) \leq h(S)$, then the maximum of the sectional curvature of $M$ is bounded below by -1 , and is equal to -1 if and only if $M$ and $S$ are isometric.

Theorem 1.3 provides the counterpart to Hamenstädt's entropy rigidity theorem under the Sullivan dictionary. Indeed, Corollary 20.8 in [BM] shows that the topological entropy $h(f)$ of an expanding Thurston map $f$ is $\log (\operatorname{deg}(f))$. Hence, the theorem provides a lower bound on the asymptotic upper curvature of an expanding Thurston map $f$ in terms of the topological entropy of $f$, i.e.

$$
K_{u}(f) \geq-\frac{1}{4} h(f)^{2}
$$

together with a condition for equality to hold.

Acknowledgements. This paper is part of the author's PhD thesis under the supervision of Mario Bonk. The author would like to thank Mario Bonk for introducing her to and teaching her about the subject of Thurston maps and its related fields. The author is inspired by his enthusiasm and mathematical wisdom, and is especially grateful for his patience and encouragement. The author would like to thank Dennis Sullivan for valuable conversations and sharing his mathematical insights. The author benefited greatly from Dick Canary's mini-course on the Kleinian group aspects of the Sullivan dictionary. The author 
also would like to thank Michael Zieve and Alan Stapledon for useful comments and feedback.

\section{Expanding Thurston maps and Cell Decompositions}

In this section we review some definitions and facts on expanding Thurston maps. We refer the reader to Section 3 in [BM] for more details. We write $\mathbb{N}$ for the set of positive integers, and $\mathbb{N}_{0}$ for the set of non-negative integers. We denote the identity map on $\mathbb{S}^{2}$ by id $\mathbb{S}^{2}$.

Let $\mathbb{S}^{2}$ be a topological 2-sphere with a fixed orientation. A continuous map $f: \mathbb{S}^{2} \rightarrow \mathbb{S}^{2}$ is called a branched covering map over $\mathbb{S}^{2}$ if $f$ can be locally written as

$$
z \mapsto z^{d}
$$

under certain orientation-preserving coordinate changes of the domain and range. More precisely, we require that for any point $p \in \mathbb{S}^{2}$, there exists some integer $d>0$, an open neighborhood $U_{p} \subseteq \mathbb{S}^{2}$ of $p$, an open neighborhood $V_{q} \subseteq \mathbb{S}^{2}$ of $q=f(p)$, and orientation-preserving homeomorphism

$$
\phi: U_{p} \rightarrow U \subseteq \mathbb{C}
$$

and

$$
\psi: V_{p} \rightarrow V \subseteq \mathbb{C}
$$

with $\phi(p)=0$ and $\psi(q)=0$ such that

$$
\left(\psi \circ f \circ \phi^{-1}\right)(z)=z^{d}
$$

for all $z \in U$. The positive integer $d=\operatorname{deg}_{f}(p)$ is called the local degree of $f$ at $p$ and only depends on $f$ and $p$. A point $p \in \mathbb{S}^{2}$ is called a critical point of $f$ if $\operatorname{deg}_{f}(p) \geq 2$, and a point $q$ is called critical value of $f$ if there is a critical point in its preimage $f^{-1}(q)$. If $f$ is a branched covering map of $\mathbb{S}^{2}, f$ is open and surjective. There are only finitely many critical points of $f$ and $f$ is finite-to-one due to the compactness of $\mathbb{S}^{2}$. Hence, $f$ is a covering map away from the critical points in the domain and critical values in the range. The degree $\operatorname{deg}(f)$ of $f$ is the cardinality of the preimage over a non-critical value. In addition, we have

$$
\operatorname{deg}(f)=\sum_{p \in f^{-1}(q)} \operatorname{deg}_{f}(p)
$$

for every $q \in \mathbb{S}^{2}$.

For $n \in \mathbb{N}$, we denote the $n$-th iterate of $f$ as

$$
f^{n}=\underbrace{f \circ f \circ \cdots \circ f}_{n \text { factors }} .
$$

We also set $f^{0}=\operatorname{id}_{\mathbb{S}^{2}}$.

If $f$ is a branched cover of $\mathbb{S}^{2}$, so is $f^{n}$, and

$$
\operatorname{deg}\left(f^{n}\right)=\operatorname{deg}(f)^{n} .
$$


Let $\operatorname{crit}(f)$ be the set of all the critical points of $f$. We define the set of postcritical points of $f$ as

$$
\operatorname{post}(f)=\bigcup_{n \in \mathbb{N}}\left\{f^{n}(c): c \in \operatorname{crit}(f)\right\} .
$$

We call a map $f$ postcritically-finite if the cardinality of post $(f)$ is finite. Notice that $f$ is postcritically-finite if and only if there is some $n \in \mathbb{N}$ for which $f^{n}$ is postcritically-finite.

Let $\mathcal{C} \subseteq \mathbb{S}^{2}$ be a Jordan curve containing post $(f)$. We fix a metric $d$ on $\mathbb{S}^{2}$ that induces the standard metric topology on $\mathbb{S}^{2}$. Denote by $\operatorname{mesh}(f, n, \mathcal{C})$ the supremum of the diameters of all connected components of the set $f^{-n}\left(\mathbb{S}^{2} \backslash \mathcal{C}\right)$.

Definition 2.1. A branched covering map $f: \mathbb{S}^{2} \rightarrow \mathbb{S}^{2}$ is called a Thurston map if $\operatorname{deg}(f) \geq 2$ and $f$ is postcritically-finite. A Thurston map $f: \mathbb{S}^{2} \rightarrow \mathbb{S}^{2}$ is called expanding if there exists a Jordan curve $\mathcal{C} \subseteq \mathbb{S}^{2}$ with $\mathcal{C} \supseteq \operatorname{post}(f)$ and

$$
\lim _{n \rightarrow \infty} \operatorname{mesh}(f, n, \mathcal{C})=0 .
$$

The relation (6) is a topological property, as it is independent of the choice of the metric, as long as the metric induces the standard topology on $\mathbb{S}^{2}$. Lemma 8.1 in [BM] shows that if the relation (6) is satisfied for one Jordan curve $\mathcal{C}$ containing post $(f)$, then it holds for every such curve. One can essentially show that a Thurston map is expanding if and only if all the connected components in the preimage under $f^{-n}$ of any open Jordan region not containing post $(f)$ become uniformly small as $n$ goes to infinity.

The following theorem (Theorem 1.2 in [BM]) says that there exists an invariant Jordan curve for some iterates of $f$.

Theorem 2.2. If $f: \mathbb{S}^{2} \rightarrow \mathbb{S}^{2}$ is an expanding Thurston map, then for some $n \in \mathbb{N}$ there exists a Jordan curve $\mathcal{C} \subseteq \mathbb{S}^{2}$ containing post $(f)$ such that $\mathcal{C}$ is invariant under $f^{n}$, i.e., $f^{n}(\mathcal{C}) \subseteq \mathcal{C}$.

Recall that an isotopy $H$ between two homeomorphisms is a homotopy so that at each time $t \in[0,1]$, the map $H_{t}$ is a homeomorphism. An isotopy $H$ relative to a set $A$ is an isotopy satisfying

$$
H_{t}(a)=H_{0}(a)=H_{1}(a)
$$

for all $a \in A$ and $t \in[0,1]$.

Definition 2.3. Consider two Thurston maps $f: \mathbb{S}^{2} \rightarrow \mathbb{S}^{2}$ and $g: \mathbb{S}_{1}^{2} \rightarrow$ $\mathbb{S}_{1}^{2}$, where $\mathbb{S}^{2}$ and $\mathbb{S}_{1}^{2}$ are 2 -spheres. We call the maps $f$ and $g$ (Thurston) equivalent if there exist homeomorphisms $h_{0}, h_{1}: \mathbb{S}^{2} \rightarrow \mathbb{S}_{1}^{2}$ that are isotopic relative to post $(f)$ such that $h_{0} \circ f=g \circ h_{1}$. We call the maps $f$ and $g$ topologically conjugate if there exists a homeomorphism $h: \mathbb{S}^{2} \rightarrow \mathbb{S}_{1}^{2}$ such that $h \circ f=g \circ h$. 
For equivalent Thurston maps, we have the following commutative diagram

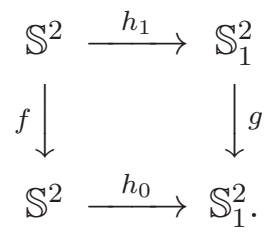

We now consider the cardinality of the postcritical set of $f$. In Remark 5.5 in $\mathrm{BM}$, it is proved that there are no Thurston maps with $\#$ post $(f) \leq 1$. Proposition 6.2 in [BM] shows that all Thurston maps with \# post $(f)=2$ are Thurston equivalent to a power map on the Riemann sphere,

$$
z \mapsto z^{k}, \text { for some } k \in \mathbb{Z} \backslash\{-1,0,1\} .
$$

Corollary 6.3 in [BM] states that if $f: \mathbb{S}^{2} \rightarrow \mathbb{S}^{2}$ is an expanding Thurston map, then \# post $(f) \geq 3$.

Let $f: \mathbb{S}^{2} \rightarrow \mathbb{S}^{2}$ be a Thurston map, and let $\mathcal{C} \subseteq \mathbb{S}^{2}$ be a Jordan curve containing post $(f)$. By the Schönflies theorem, the set $\mathbb{S}^{2} \backslash \mathcal{C}$ has two connected components, which are both homeomorphic to the open unit disk. Let $T_{0}$ and $T_{0}^{\prime}$ denote the closures of these components. They are cells of dimension 2 , which we call 0 -tiles. The postcritical points of $f$ are called 0 -vertices of $T_{0}$ and $T_{0}^{\prime}$, singletons of which are cells of dimension 0 . We call the closed arcs between vertices 0 -edges of $T_{0}$ and $T_{0}^{\prime}$, which are cells of dimension 1 . These 0 -vertices, 0 -edges and 0 -tiles form a cell decomposition of $\mathbb{S}^{2}$, denoted by $\mathcal{D}^{0}=\mathcal{D}^{0}(f, \mathcal{C})$. We call the elements in $\mathcal{D}^{0} 0$-cells. Let $\mathcal{D}^{1}=\mathcal{D}^{1}(f, \mathcal{C})$ be the set of connected subsets $c \subseteq \mathbb{S}^{2}$ such that $f(c)$ is a cell in $\mathcal{D}^{0}$ and $\left.f\right|_{c}$ is a homeomorphism of $c$ onto $f(c)$. Call $c$ a 1-tile if $f(c)$ is a 0-tile, call $c$ a 1-edge if $f(c)$ is a 0-edge, and call $c$ a 1-vertex if $f(c)$ is a 1 -vertex. Lemma 5.4 in $\left[\mathrm{BM}\right.$ states that $\mathcal{D}^{1}$ is a cell decomposition of $\mathbb{S}^{2}$. Continuing in this manner, let $\mathcal{D}^{n}=\mathcal{D}^{n}(f, \mathcal{C})$ be the set of all connected subsets of $c \subseteq \mathbb{S}^{2}$ such that $f(c)$ is a cell in $\mathcal{D}^{n-1}$ and $\left.f\right|_{c}$ is a homeomorphism of $c$ onto $f(c)$, and call these connected subsets $n$-tiles, $n$-edges and $n$-vertices correspondingly, for $n \in \mathbb{N}_{0}$. By Lemma 5.4 in [BM], $\mathcal{D}^{n}$ is a cell decomposition of $\mathbb{S}^{2}$, for each $n \in \mathbb{N}_{0}$, and we call the elements in $\mathcal{D}^{n} n$-cells. The following lemma lists some properties of these cell decompositions. For more details, we refer the reader to Proposition 6.1 in $[\mathrm{BM}]$.

Lemma 2.4. Let $k, n \in \mathbb{N}_{0}$, let $f: \mathbb{S}^{2} \rightarrow \mathbb{S}^{2}$ be a Thurston map, let $\mathcal{C} \subset \mathbb{S}^{2}$ be a Jordan curve with $\mathcal{C} \supseteq \operatorname{post}(f)$, and let $m=\# \operatorname{post}(f)$.

(1) If $\tau$ is any $(n+k)$-cell, then $f^{k}(\tau)$ is an $n$-cell, and $\left.f^{k}\right|_{\tau}$ is a homeomorphism of $\tau$ onto $f^{k}(\tau)$.

(2) Let $\sigma$ be an $n$-cell. Then $f^{-k}(\sigma)$ is equal to the union of all $(n+k)$-cells $\tau$ with $f^{k}(\tau)=\sigma$. 
(3) The number of $n$-vertices is less than or equal to $m \operatorname{deg}(f)^{n}$, the number of $n$-edges is $m \operatorname{deg}(f)^{n}$, and the number of $n$-tiles is $2 \operatorname{deg}(f)^{n}$.

(4) The n-edges are precisely the closures of the connected components of $f^{-n}(\mathcal{C}) \backslash f^{-n}(\operatorname{post}(f))$. The $n$-tiles are precisely the closures of the connected components of $\mathbb{S}^{2} \backslash f^{-n}(\mathcal{C})$.

(5) Every $n$-tile is an $m$-gon, i.e., the number of $n$-edges and $n$ vertices contained in its boundary is equal to $\mathrm{m}$.

We obtain a sequence of cell decompositions of $\mathbb{S}^{2}$ from a Thurston map and a Jordan curve on $\mathbb{S}^{2}$. It would be nice if the local degrees of the map $f$ at all the vertices were bounded, and this can be obtained by the assumption of no periodic critical points (see [BM, Lemma 16.1]).

Lemma 2.5. Let $f: \mathbb{S}^{2} \rightarrow \mathbb{S}^{2}$ be a branched covering map. Then $f$ has no periodic critical points if and only if there exists $N \in \mathbb{N}$ such that

$$
\operatorname{deg}_{f^{n}}(p) \leq N
$$

for all $p \in \mathbb{S}^{2}$ and all $n \in \mathbb{N}$.

Henceforth we assume that all Thurston maps have no periodic critical points.

Let $f: \mathbb{S}^{2} \rightarrow \mathbb{S}^{2}$ be an expanding Thurston map and let $\mathcal{C}$ be a Jordan curve containing post $(f)$.

Definition 2.6. A set $K \subseteq \mathbb{S}^{2}$ joins opposite sides of $\mathcal{C}$ if $\# \operatorname{post}(f) \geq 4$ and $K$ meets two disjoint 0 -edges, or if \#post $(f)=3$ and $K$ meets all three 0-edges.

Let $D_{n}=D_{n}(f, \mathcal{C})$ be the minimum number of $n$-tiles needed to join opposite sides of a Jordan curve $\mathcal{C}$. More precisely,

(7) $D_{n}=\min \left\{N \in \mathbb{N}\right.$ : there exist $n$-tiles $X_{1}, \ldots, X_{N}$ such that

$$
\left.\bigcup_{j=1}^{N} X_{j} \text { is connected and joins opposite sides of } \mathcal{C}\right\} \text {. }
$$

Of course, $D_{n}$ depends on $f$ and $\mathcal{C}$.

Let $f$ be an expanding Thurston map. For any two Jordan curves $\mathcal{C}$ and $\mathcal{C}^{\prime}$ with post $(f) \subset \mathcal{C}, \mathcal{C}^{\prime}$, inequality (17.1) in [BM] states that there exists a constant $c>0$ such that for all $n>0$,

$$
\frac{1}{c} D_{n}(f, \mathcal{C}) \leq D_{n}\left(f, \mathcal{C}^{\prime}\right) \leq c D_{n}(f, \mathcal{C}) .
$$

Proposition 17.1 in $[\mathrm{BM}]$ says that:

Proposition 2.7. For an expanding Thurston map $f: \mathbb{S}^{2} \rightarrow \mathbb{S}^{2}$, and a Jordan curve $\mathcal{C}$ containing $\operatorname{post}(f)$, the limit

$$
\Lambda_{0}=\Lambda_{0}(f):=\lim _{n \rightarrow \infty} D_{n}(f, \mathcal{C})^{1 / n}
$$

exists and is independent of $\mathcal{C}$. 
We call $\Lambda_{0}(f)$ the combinatorial expansion factor of $f$.

Proposition 17.2 in $[\mathrm{BM}$, states that:

Proposition 2.8. If $f: \mathbb{S}^{2} \rightarrow \mathbb{S}^{2}$ and $g: \mathbb{S}_{1}^{2} \rightarrow \mathbb{S}_{1}^{2}$ are expanding Thurston maps that are topologically conjugate, then $\Lambda_{0}(f)=\Lambda_{0}(g)$.

Definition 2.9. Let $f: \mathbb{S}^{2} \rightarrow \mathbb{S}^{2}$ be an expanding Thurston map, and let $\mathcal{C} \subseteq \mathbb{S}^{2}$ be a Jordan curve containing post $(f)$. Let $x, y \in \mathbb{S}^{2}$. For $x \neq y$ we define

$$
\begin{array}{r}
m_{f, \mathcal{C}}(x, y)=\min \left\{n \in \mathbb{N}_{0}: \text { there exist disjoint } n \text {-tiles } X \text { and } Y\right. \\
\text { for }(f, \mathcal{C}) \text { with } x \in X \text { and } y \in Y\} .
\end{array}
$$

If $x=y$, we define $m_{f, \mathcal{C}}(x, x)=\infty$.

The minimum in the definition above is always obtained since the diameters of $n$-tiles go to 0 as $n \rightarrow \infty$. We usually drop one or both subscripts in $m_{f, \mathcal{C}}(x, y)$ if $f$ or $\mathcal{C}$ is clear from the context. If we define for $x, y \in \mathbb{S}^{2}$ and $x \neq y$, $m_{f, \mathcal{C}}^{\prime}(x, y)=\max \left\{n \in \mathbb{N}_{0}\right.$ : there exist nondisjoint $n$-tiles $X$ and $Y$

$$
\text { for }(f, \mathcal{C}) \text { with } x \in X \text { and } y \in Y\} \text {, }
$$

then $m_{f, \mathcal{C}}$ and $m_{f, \mathcal{C}}^{\prime}$ are essentially the same up to a constant (see Lemma $8.6(\mathrm{v})$ in $[\mathrm{BM}])$.

Lemma 2.10. Let $m_{f, \mathcal{C}}$ and $m_{f, \mathcal{C}}^{\prime}$ as defined above. There exists a constant $k>0$, such that for any $x, y \in \mathbb{S}^{2}$ and $x \neq y$,

$$
m_{f, \mathcal{C}}(x, y)-k \leq m_{f, \mathcal{C}}^{\prime}(x, y) \leq m_{f, \mathcal{C}}(x, y)+1 .
$$

Definition 2.11. Let $f: \mathbb{S}^{2} \rightarrow \mathbb{S}^{2}$ be an expanding Thurston map and $d$ be a metric on $\mathbb{S}^{2}$. The metric $d$ is called a visual metric for $f$ if there exists a Jordan curve $\mathcal{C} \subseteq \mathbb{S}^{2}$ containing post $(f)$, constants $\Lambda>1$ and $C \geq 1$ such that

$$
\frac{1}{C} \Lambda^{-m_{f, \mathcal{C}}(x, y)} \leq d(x, y) \leq C \Lambda^{-m_{f, \mathcal{C}}(x, y)}
$$

for all $x, y \in \mathbb{S}^{2}$.

Proposition 8.9 in [BM] states that for any expanding Thurston map $f: \mathbb{S}^{2} \rightarrow \mathbb{S}^{2}$, there exists a visual metric for $f$, which induces the standard topology on $\mathbb{S}^{2}$. Lemma 8.10 in the same paper gives the following characterization of visual metrics.

Lemma 2.12. Let $f: \mathbb{S}^{2} \rightarrow \mathbb{S}^{2}$ be an expanding Thurston map. Let $\mathcal{C} \subseteq \mathbb{S}^{2}$ be a Jordan curve containing post $(f)$, and $d$ be a visual metric for $f$ with expansion factor $\Lambda>1$. Then there exists a constant $C>1$ such that

(1) $d(\sigma, \tau) \geq(1 / C) \Lambda^{-n}$ whenever $\sigma$ and $\tau$ are disjoint $n$-cells,

(2) $(1 / C) \Lambda^{-n} \leq \operatorname{diam}(\tau) \leq C \Lambda^{-n}$ for $\tau$ as any $n$-edge or $n$-tile. 
Conversely, if $d$ is a metric on $\mathbb{S}^{2}$ satisfying conditions (1) and (2) for some constant $C>1$, then $d$ is a visual metric with expansion factor $\Lambda>1$.

The combinatorial expansion factor $\Lambda_{0}(f)$ (defined after Proposition 2.7) is the supremum of the expansion factors for all the visual metric for $f$ (see Theorem 1.7 in $[\mathrm{BM}]$ ).

Theorem 2.13. Let $f: \mathbb{S}^{2} \rightarrow \mathbb{S}^{2}$ be an expanding Thurston map with combinatorial expansion factor $\Lambda_{0}(f)$. Then

$$
\begin{array}{r}
\Lambda_{0}:=\sup \{\Lambda>1: \quad \text { there exists a visual metric for } f \\
\text { with expansion factor } \Lambda\} .
\end{array}
$$

\section{Gromov Hyperbolic Spaces}

In this section, we review the definitions of Gromov hyperbolic spaces and the asymptotic upper curvature for Gromov hyperbolic spaces.

Let us first review some basic facts about Gromov hyperbolic spaces. We refer the reader to $\mathrm{BS}$ as a general source on Gromov hyperbolic spaces. Let $(X, d)$ be a geodesic metric space. For any points $x, y, p \in$ $X$, the Gromov product $(x, y)_{p}$ of $x$ and $y$ with respect to base point $p$ is defined as

$$
(x, y)_{p}:=\frac{1}{2}[d(x, p)+d(y, p)-d(x, y)] .
$$

The space $X$ is called $\delta$-hyperbolic (or Gromov hyperbolic) for some $\delta \geq 0$ if there exists a base point $p \in X$, such that for all $x, y, z \in X$ we have

$$
(x, y)_{p} \geq \min \left\{(x, z)_{p},(z, y)_{p}\right\}-\delta .
$$

If this inequality holds for some base point $p \in X$, then it also holds for any other $p^{\prime} \in X$ with $\delta$ being replaced by $2 \delta$.

Let $(X, d)$ be a Gromov hyperbolic metric space with a fixed base point $p \in X$. A sequence of points $\left\{x_{i}\right\} \subseteq X$ converges to infinity if

$$
\lim _{i, j \rightarrow \infty}\left(x_{i}, x_{j}\right)_{p}=\infty .
$$

This property of a sequence $\left\{x_{i}\right\}$ does not depend on the base point $p \in X$. We say two sequences converging to infinity $\left\{x_{i}\right\}$ and $\left\{x_{i}^{\prime}\right\}$ are equivalent if

$$
\lim _{i \rightarrow \infty}\left(x_{i}, x_{i}^{\prime}\right)_{p}=\infty
$$

The boundary at infinity $\partial_{\infty} X$ of $X$ is defined to be the set of equivalence classes of sequences of points converging to infinity. One can also define the Gromov product for points $\xi, \xi^{\prime} \in \partial_{\infty} X$ and $p \in X$ as

$$
\left(\xi, \xi^{\prime}\right)_{p}:=\inf \liminf _{i \rightarrow \infty}\left(x_{i}, x_{i}^{\prime}\right)_{p}
$$


where the infimum is taken over all sequences $\left\{x_{i}\right\} \in \xi$ and $\left\{x_{i}^{\prime}\right\} \in \xi^{\prime}$. Here $\left(\xi, \xi^{\prime}\right)_{p}=\infty$ if and only if $\xi=\xi^{\prime}$.

A metric $\rho$ on the boundary at infinity $\partial_{\infty} X$ of a Gromov hyperbolic space $X$ is called visual if there exist $p \in X, \Lambda>1$ and $k \geq 1$ such that for all $\xi, \xi^{\prime} \in \partial_{\infty} X$, we have that

$$
\frac{1}{k} \Lambda^{-\left(\xi, \xi^{\prime}\right)_{p}} \leq \rho\left(\xi, \xi^{\prime}\right) \leq k \Lambda^{-\left(\xi, \xi^{\prime}\right)_{p}} .
$$

We call the constant $\Lambda$ in this inequality the expansion factor of the visual metric $\rho$. Recall that we also defined a visual metric for an expanding Thurston map (see Definition 2.11). When it is not clear from context, we will refer to the visual metric defined in (10) as a 'visual metric in the Gromov hyperbolic sense'.

Given two metric spaces $\left(X, d_{X}\right)$ and $\left(Y, d_{Y}\right)$, a map $f: X \rightarrow Y$ is called a quasi-isometry if there are constants $\lambda \geq 1$ and $k \geq 0$ such that for all $x, x^{\prime} \in X$

$$
\frac{1}{\lambda} d_{X}\left(x, x^{\prime}\right)-k \leq d_{Y}\left(f(x), f\left(x^{\prime}\right)\right) \leq \lambda d_{X}\left(x, x^{\prime}\right)+k
$$

and for all $y \in Y$,

$$
\inf _{x \in X} d_{Y}(f(x), y) \leq k .
$$

If $\lambda=1$, we call the map $f$ a rough-isometry. We say that the spaces $X$ and $Y$ are quasi-isometric (rough-isometric) if there is a quasi-isometry (rough-isometry) between them.

In $[\mathrm{BF}]$, Bonk and Foertsch introduced the notion of upper curvature bounds for Gromov hyperbolic spaces up to rough-isometry (see $[\mathrm{BF}$, Definition 1.1 and 1.2]).

Definition 3.1. Let $\kappa \in[-\infty, 0)$. We call a metric space $X$ an $A C_{u}(\kappa)$ space if there exists $p \in X$ and a constant $c \geq 0$ such that for all $x, x^{\prime} \in X$ and all finite sequences $x_{0}=x, x_{1}, \ldots, x_{n}=x^{\prime}$ in $X$ with $n>0$,

$$
\left(x, x^{\prime}\right)_{p} \geq \min _{i=1, \ldots, n}\left(x_{i-1}, x_{i}\right)_{p}-\frac{1}{\sqrt{-\kappa}} \log n-c .
$$

Here we use the convention $1 / \sqrt{\infty}=0$. We call

$$
K_{u}(X):=\inf \left\{\kappa: X \text { is an } \mathrm{AC}_{u}(\kappa) \text {-space } \in[-\infty, 0)\right\}
$$

the asymptotic upper curvature of $X$.

Rough-isometric Gromov hyperbolic spaces have the same asymptotic upper curvature since under rough-isometries, Gromov products only change by a fixed additive amount, which can be absorbed in the constant $c$ in (11).

The asymptotic upper curvature is related to the expansion factors of visual metrics in Gromov hyperbolic spaces, due to the following theorem [BF, Theorem 1.5]. 
Theorem 3.2. Let $X$ be a Gromov hyperbolic metric space. If there exists a visual metric on $\partial_{\infty} X$ with expansion factor $\Lambda>1$, then $X$ is an $A C_{u}(\kappa)$-space with $\kappa=-\log ^{2} \Lambda$. Conversely, if $X$ is an $A C_{u}(\kappa)$ space, then for every $1<\Lambda<e^{\sqrt{-\kappa}}$, there exists a visual metric on $\partial_{\infty} X$ with expansion $\Lambda$. In particular,

$$
K_{u}(X)=-\log ^{2} \Lambda_{0}
$$

where

$$
\begin{gathered}
\Lambda_{0}:=\sup \left\{\Lambda>1: \quad \text { there exists a visual metric on } \partial_{\infty} X\right. \\
\text { with expansion factor } \Lambda\} .
\end{gathered}
$$

\section{Tile GraphS}

In this section, we construct graphs for expanding Thurston maps. We prove that these graphs are Gromov hyperbolic and their boundary at infinity can be identified with $\mathbb{S}^{2}$. This construction should be compared to the construction of graphs associated to finite branched coverings in Section 3.2 and 3.3 in [HP].

Let $f: \mathbb{S}^{2} \rightarrow \mathbb{S}^{2}$ be an expanding Thurston map, and $\mathcal{C} \subset \mathbb{S}^{2}$ be a Jordan curve such that post $(f) \subset \mathcal{C}$. Recall that there is a natural sequence of cell decompositions $\mathcal{D}^{n}(f, \mathcal{C})$ on $\mathbb{S}^{2}$ whose 1 -skeletons are the pull-backs of the Jordan curve $\mathcal{C}$ under $f^{n}$ (see Section 2). Proposition 8.9 in $[\mathrm{BM}]$ states that there exists a visual metric $d$ for $f$ with expansion factor $\Lambda$ for some $\Lambda>1$.

We define a graph by the cell decompositions of $(f, \mathcal{C})$ as follows. Let

$$
V=V(f, \mathcal{C})
$$

be the set of all tiles in the cell decompositions $\mathcal{D}^{n}(f, \mathcal{C})$ of $(f, \mathcal{C})$ for $n \geq-1$, where $\mathcal{D}^{-1}(f, \mathcal{C})$ contains a single $(-1)$-tile $\mathbb{S}^{2}$. Let $V$ be the set of vertices of the graph. Define the edge set $E$ as follows: there is an edge between the two vertices $X^{n}, Y^{m} \in V$, which we indicate by the notation $X^{n} \sim Y^{m}$ if for the underlying tiles we have

$$
|n-m| \leq 1 \text { and } X^{n} \cap Y^{m} \neq \emptyset .
$$

We call the graph

$$
\mathcal{G}(f, \mathcal{C}):=G(V, E)
$$

the tile graph of $(f, \mathcal{C})$. We usually drop one or both parameters in $\mathcal{G}(f, \mathcal{C})$ if $f$ or $\mathcal{C}$ are clear from the context. We call

$$
\ell: V \rightarrow \mathbb{Z}
$$

the level function, where for an $n$-tile $X^{n}$, we have $\ell\left(X^{n}\right)=n$.

If $X \cap Y=\emptyset$, let

$\bar{m}_{f, \mathcal{C}}(X, Y):=\max \left\{m \in \mathbb{N}_{-1}:\right.$ there exist non-disjoint $m$-tiles $X^{m}$ and $Y^{m}$, such that $\left.X \cap X^{m} \neq \emptyset, Y \cap Y^{m} \neq \emptyset\right\}$; 
if $X \cap Y \neq \emptyset$, let

$$
\bar{m}_{f, \mathcal{C}}(X, Y):=\infty \text {. }
$$

Here we assume that the $\infty$-tile is the empty set. For $X, Y \in \mathcal{G}$, define

$$
m(X, Y)=m_{f, \mathcal{C}}(X, Y)=\min \left\{\ell(X), \ell(Y), \bar{m}_{f, \mathcal{C}}(X, Y)\right\}
$$

The tile graph $\mathcal{G}$ is path connected since any tile can be connected to the $(-1)$-tile $\mathbb{S}^{2}$. We give $\mathcal{G}$ the path metric $\eta$. Notice that $\mathcal{G}$ is a geodesic space under this metric. The distance of $X \in V$ to the base point $\mathbb{S}^{2}$ is

$$
\eta\left(X, \mathbb{S}^{2}\right)=\ell(X)+1
$$

For $X, Y \in \mathcal{G}$, we let

$$
\begin{aligned}
(X, Y) & :=(X, Y)_{X^{-1}}=(X, Y)_{\mathbb{S}^{2}} \\
& =1 / 2\left[\eta\left(X, \mathbb{S}^{2}\right)+\eta\left(Y, \mathbb{S}^{2}\right)-\eta(X, Y)\right] \\
& =1 / 2[\ell(X)+\ell(Y)-\eta(X, Y)]+1,
\end{aligned}
$$

be the Gromov product of $X$ and $Y$ with respect to $X^{-1}=\mathbb{S}^{2}$.

In the following, we are going to prove that the tile graph $\mathcal{G}$ equipped with the path metric $\eta$ is a Gromov hyperbolic space.

Lemma 4.1. There exists a constant $C>1$ such that for any tiles $X, Y \in \mathcal{G}$,

$$
\frac{1}{C} \Lambda^{-m(X, Y)} \leq \operatorname{diam}(X \cup Y) \leq C \Lambda^{-m(X, Y)} .
$$

Here and in the following, the diameter function $\operatorname{diam}(\cdot)$ is with respect to the visual metric $d$ on $\mathbb{S}^{2}$.

Proof. Let $m=m(X, Y)$, and let $X^{m}, Y^{m}$ be non-disjoint $m$-tiles such that

$$
X \cap X^{m} \neq \emptyset \text { and } Y \cap Y^{m} \neq \emptyset \text {. }
$$

We have that

$$
\begin{aligned}
\operatorname{diam}(X \cup Y) & \leq \operatorname{diam}(X)+\operatorname{diam}\left(X^{m}\right)+\operatorname{diam}\left(Y^{m}\right)+\operatorname{diam}(Y) \\
& \leq 4 C^{\prime} \Lambda^{-m}
\end{aligned}
$$

where $C^{\prime}>1$ is the same as the constant in Lemma 2.12, which only depends on $f$ and $\mathcal{C}$. Let $\bar{m}=\bar{m}_{f, \mathcal{C}}(X, Y)$, and let $X^{\bar{m}+1}, Y^{\bar{m}+1}$ be disjoint $(\bar{m}+1)$-tiles such that

$$
X \cap X^{\bar{m}+1} \neq \emptyset, \quad Y \cap Y^{\bar{m}+1} \neq \emptyset .
$$


Then

$$
\begin{aligned}
\operatorname{diam}(X \cup Y) & \geq \max \left\{\operatorname{diam}(X), \operatorname{diam}(Y), d\left(X^{\bar{m}+1}, Y^{\bar{m}+1}\right)\right\} \\
& \geq \frac{1}{C^{\prime}} \max \left\{\Lambda^{-\ell(X)}, \Lambda^{-\ell(Y)}, \Lambda^{-\bar{m}}\right\} \\
& \geq \frac{1}{C^{\prime}} \Lambda^{-\min \{\ell(X), \ell(Y), \bar{m}\}} \\
& =\frac{1}{C^{\prime}} \Lambda^{-m}
\end{aligned}
$$

where $C^{\prime}>1$ is the same $C$ as in Lemma 2.12, which only depends on $f$ and $\mathcal{C}$. Let $C=4 C^{\prime}$, and the lemma follows.

Lemma 4.2. There exists a constant $k \geq 1$ such that for any tiles $X, Y \in \mathcal{G}$,

$$
\operatorname{diam}(X \cup Y) \leq k \Lambda^{-(X, Y)} .
$$

Proof. Let $\eta=\eta(X, Y)$. Pick any path $X_{0}=X, X_{1}, \ldots, X_{\eta}=Y$. Then

$$
\begin{aligned}
\operatorname{diam}(X \cup Y) & \leq \sum_{i=0}^{\eta} \operatorname{diam}\left(X_{i}\right) \\
& \leq C \sum_{i=0}^{\eta} \Lambda^{-\ell\left(X_{i}\right)} \\
& \leq C \min _{0 \leq l \leq \eta}\left\{\sum_{i=0}^{l} \Lambda^{-\ell(X)+i}+\sum_{i=l+1}^{\eta} \Lambda^{-\ell(Y)+(\eta-i)}\right\} \\
& \leq \frac{C \Lambda}{\Lambda-1} \min _{0 \leq l \leq \eta}\left\{\Lambda^{-\ell(X)+l}+\Lambda^{-\ell(Y)+(\eta-l)}\right\} .
\end{aligned}
$$

Notice that on the right hand-side the minimum is obtained when the two exponents of $\Lambda$ are the same:

$$
-\ell(X)+l=-\ell(Y)+(\eta-l),
$$

so we let

$$
l=\left[\frac{1}{2}(\ell(X)-\ell(Y)+\eta)\right]
$$

be the integer part of $\frac{1}{2}(\ell(X)-\ell(Y)+\eta)$. Hence, we have

$$
\begin{aligned}
\operatorname{diam}(X \cup Y) & \leq \frac{2 C \Lambda}{\Lambda-1} \Lambda^{-[1 / 2(\ell(X)+\ell(Y)-\eta)]} \\
& \leq k \Lambda^{-(X, Y)}
\end{aligned}
$$

where $C>1$ is the same $C$ as in Lemma 2.12, and

$$
k=\frac{2 C \Lambda^{3}}{\Lambda-1}
$$

also only depends on $f$. 
Proposition 4.3. There exists a constant $C^{\prime}>0$, such that for any tiles $X, Y \in \mathcal{G}$,

$$
m(X, Y)-1 \leq(X, Y) \leq m(X, Y)+C^{\prime} .
$$

Proof. By Lemma 4.1 and Lemma 4.2, we have that

$$
\frac{1}{C} \Lambda^{-m(X, Y)} \leq \operatorname{diam}(X \cup Y) \leq k \Lambda^{-(X, Y)}
$$

for some constants $C, k>1$ which only depend on $f$. Hence, there exists a constant $C^{\prime}>0$, such that for any tiles $X, Y \in \mathcal{G}$,

$$
(X, Y) \leq m(X, Y)+C^{\prime} .
$$

For the other inequality, let $m=m(X, Y)$, and let $X^{m}, Y^{m}$ be nondisjoint $m$-tiles such that

$$
X \cap X^{m} \neq \emptyset, \quad Y \cap Y^{m} \neq \emptyset .
$$

So

$$
\eta\left(X, X^{m}\right) \leq \ell(X)-m+1
$$

and

$$
\eta\left(Y, Y^{m}\right) \leq \ell(Y)-m+1 .
$$

By the triangle inequality, we have that

$$
\begin{aligned}
\eta(X, Y) & \leq \eta\left(X, X^{m}\right)+\eta\left(X^{m}, Y\right) \\
& \leq \eta\left(X, X^{m}\right)+\eta\left(Y^{m}, Y\right)+1 \\
& \leq(\ell(X)-m)+(\ell(Y)-m)+3 .
\end{aligned}
$$

Hence, we obtain that

$$
\begin{aligned}
(X, Y) & =(X, Y)_{X^{-1}}=1 / 2[\ell(X)+\ell(Y)-\eta(X, Y)]+1 \\
& \geq 1 / 2[\ell(X)+\ell(Y)-(\ell(X)-m)-(\ell(Y)-m)-3]+1 \\
& \geq m(X, Y)-1 .
\end{aligned}
$$

Lemma 4.4. There exists a number $c \geq 0$ such that for any tiles $X, Y, Z \in \mathcal{G}$,

$$
m(X, Y) \geq \min \{m(X, Z), m(Y, Z)\}-c .
$$

Proof. For any $X, Y, Z \in \mathcal{G}$,

$$
\begin{aligned}
\operatorname{diam}(X \cup Y)= & \max \left\{d(x, y), d\left(x, x^{\prime}\right), d\left(y, y^{\prime}\right): x, x^{\prime} \in X, y, y^{\prime} \in Y\right\} \\
\leq & \max \left\{d(x, z)+d(z, y), d\left(x, x^{\prime}\right), d\left(y, y^{\prime}\right):\right. \\
& \left.x, x^{\prime} \in X, y, y^{\prime} \in Y, z \in Z\right\} \\
\leq & \max \left\{d(x, z), d\left(x, x^{\prime}\right): x, x^{\prime} \in X, z \in Z\right\} \\
& +\max \left\{d(z, y), d\left(y, y^{\prime}\right): y, y^{\prime} \in Y, z \in Z\right\} \\
\leq & \operatorname{diam}(X \cup Z)+\operatorname{diam}(Z \cup Y),
\end{aligned}
$$


and so

$$
\operatorname{diam}(X \cup Y) \leq 2 \max \{\operatorname{diam}(X \cup Z), \operatorname{diam}(Z \cup Y)\} .
$$

By Lemma 4.1, there exists a constant $k>1$, such that for any $X, Y \in \mathcal{G}$,

$$
\frac{1}{k} \Lambda^{-m(X, Y)} \leq \operatorname{diam}(X \cup Y) \leq k \Lambda^{-m(X, Y)} .
$$

Hence, by the inequalities above and inequality (14), we have that

$$
\begin{aligned}
m(X, Y) & \geq-\log _{\Lambda}(k \operatorname{diam}(X \cup Y)) \\
& \geq-\log _{\Lambda}(2 k \max \{\operatorname{diam}(X \cup Z), \operatorname{diam}(Z \cup Y)\}) \\
& \geq \min \left\{-\log _{\Lambda}(2 k \operatorname{diam}(X \cup Z)),-\log _{\Lambda}(2 k \operatorname{diam}(Z \cup Y))\right\} \\
& \geq \min \{m(X, Z), m(Y, Z)\}-c
\end{aligned}
$$

for some $c \geq 0$ that only depends on $f$.

Theorem 4.5. Let $f: \mathbb{S}^{2} \rightarrow S^{2}$ be an expanding Thurston map and let $\mathcal{C} \subset \mathbb{S}^{2}$ be a Jordan curve containing post $(f)$. Then the tile graph $\mathcal{G}(f, \mathcal{C})$ equipped with the path metric $\eta$ is a Gromov hyperbolic space.

Proof. For any tiles $X, Y \in \mathcal{G}$, by Proposition 4.3, the Gromov product $(X, Y)$ defined in equation (13) is equal to $m(X, Y)$ up to a constant which only depends on $f$. So by Lemma 4.4, there exists a constant $c^{\prime}>0$, such that for any tiles $X, Y, Z \in \mathcal{G}$,

$$
(X, Y) \geq \min \{(X, Z),(Y, Z)\}-c^{\prime} .
$$

Therefore, the graph $G(f, \mathcal{C})$ equipped with the path metric $\eta$ is a Gromov hyperbolic space.

Remark. In the proofs of Proposition 4.3 and Lemma 4.4, we used visual metrics as a bridge to connect $m(\cdot, \cdot)$ and the Gromov product $(\cdot, \cdot)$. This idea is contained in $[\mathrm{BP}$. Theorem 4.5, Proposition 4.3 and Lemma 4.4 can also be proved combinatorially without using visual metrics.

Proposition 4.6. For any Jordan curves $\mathcal{C}$ and $\mathcal{C}^{\prime}$ containing post $(f)$, the tile graphs $\mathcal{G}=\mathcal{G}(f, \mathcal{C})$ and $\mathcal{G}^{\prime}=\mathcal{G}\left(f, \mathcal{C}^{\prime}\right)$ equipped with path metric respectively are rough-isometric.

Proof. By equation (13), for any $X, Y \in \mathcal{G}(f, \mathcal{C})$, we have

$$
\eta(X, Y)=\ell(X)+\ell(Y)+2-2(X, Y) .
$$

We have similar relations for the path metric $\eta^{\prime}$ of $\mathcal{G}^{\prime}$. Let $m=m_{f, \mathcal{C}}$ and $m^{\prime}=m_{f, \mathcal{C}^{\prime}}$ as defined in equation (12). We know that $m(X, Y)$ and $(X, Y)$ are equal up to a constant that only depends on $f$ by Proposition 4.3. So if we can show that there exists a level-preserving bijection $g: \mathcal{G} \rightarrow \mathcal{G}^{\prime}$ and a constant $\lambda \geq 0$, such that for any $X, Y \in \mathcal{G}$,

$$
m(X, Y)-\lambda \leq m^{\prime}(g(X), g(Y)) \leq m(X, Y)+\lambda,
$$


then by equation (15), the map $g$ will be a rough isometry between the path metrics of $\mathcal{G}$ and $\mathcal{G}^{\prime}$.

Fix $p \in \operatorname{post}(f)$. We will define

$$
g: \mathcal{G}(f, \mathcal{C}) \rightarrow \mathcal{G}\left(f, \mathcal{C}^{\prime}\right)
$$

by specifying a bijection between $n$-tiles of $(f, \mathcal{C})$ and $\left(f, \mathcal{C}^{\prime}\right)$ for all $n \geq-1$. For $n=-1$, let

$$
g\left(\mathbb{S}^{2}\right)=\mathbb{S}^{2} .
$$

For $n \geq 0$, and for any $q \in f^{-n}(p)$, we claim that there exists a bijection $g_{n, q}$ between $n$-tiles of $(f, \mathcal{C})$ containing $q$ and $n$-tiles of $\left(f, \mathcal{C}^{\prime}\right)$ containing $q$,

$g_{n, q}:\left\{n\right.$-tile $\left.X \in \mathcal{D}^{n}(f, \mathcal{C}): q \in X\right\} \rightarrow\left\{n\right.$-tile $\left.X^{\prime} \in \mathcal{D}^{n}\left(f, \mathcal{C}^{\prime}\right): q \in X^{\prime}\right\}$.

Indeed, the number of tiles containing $q$ is equal to twice the degree of $f^{n}$ at $q$, and this justifies the existence of the bijection $g_{n, q}$. Since every $n$-tile contains exactly one point in $f^{-n}(p)$, we get a bijection of all $n$-tiles by $g_{n, q}$ for $q \in f^{-n}(p)$.

For any $X, Y \in \mathcal{G}$, let $X^{\prime}, Y^{\prime} \in \mathcal{G}^{\prime}$ be their images under $g$. It follows from the definition of $g$ that

$$
X \cap X^{\prime} \neq \emptyset \text { and } Y \cap Y^{\prime} \neq \emptyset .
$$

Now we are going to show that there exists $k \geq 1$, such that for any $X, Y \in \mathcal{G}$,

$$
\frac{1}{k} \operatorname{diam}\left(X^{\prime} \cup Y^{\prime}\right) \leq \operatorname{diam}(X \cup Y) \leq k \operatorname{diam}\left(X^{\prime} \cup Y^{\prime}\right) .
$$

Let $m=m(X, Y)$. We have that

$$
\begin{aligned}
\operatorname{diam}(X \cup Y) & \leq \operatorname{diam}(X)+\operatorname{diam}\left(X^{\prime} \cup Y^{\prime}\right)+\operatorname{diam}(Y) \\
& \leq C^{2} \operatorname{diam}\left(X^{\prime}\right)+C^{2} \operatorname{diam}\left(Y^{\prime}\right)+\operatorname{diam}\left(X^{\prime} \cup Y^{\prime}\right) \\
& \leq\left(C^{2}+1\right) \operatorname{diam}\left(X^{\prime} \cup Y^{\prime}\right),
\end{aligned}
$$

where $C>1$ is the same $C$ as in Lemma 2.12, which only depends on $f, \mathcal{C}$ and $\mathcal{C}^{\prime}$. This implies that

$$
\operatorname{diam}(X \cup Y) \leq k \operatorname{diam}\left(X^{\prime} \cup Y^{\prime}\right),
$$

for some $k>1$ only depending on $f$. Similarly, we get that

$$
\operatorname{diam}\left(X^{\prime} \cup Y^{\prime}\right) \leq k \operatorname{diam}(X \cup Y) .
$$

Since $\operatorname{diam}(X \cup Y)$ and $\Lambda^{-m(X, Y)}$ are the same up to a scaling by Lemma 4.1, there exists a constant $\lambda>0$, such that

$$
m(X, Y)-\lambda \leq m^{\prime}(g(X), g(Y)) \leq m(X, Y)+\lambda
$$

for all $X, Y \in \mathcal{G}(f, \mathcal{C})$.

Remark. In the proof of Proposition 4.6, the bijective rough-isometry $g$ between tile graphs of two different Jordan curves induces a bijection $g_{\infty}$ on the boundary at infinity of these two tile graphs. 
Remark. Theorem 4.5 and Proposition 4.6 should be compared to Theorem 3.3.1 in [HP]. More specifically, Haïssinsky and Pilgrim introduce a collection of graphs for a more general notion of an expanding Thurston map and prove that they are Gromov hyperbolic, and quasiisometric to each other. One should be able to prove that the tile graph $\mathcal{G}(f, \mathcal{C})$ is quasi-isometric to a graph from Theorem 3.3.1 in [HP], and hence deduce an alternative proof of Theorem 4.5.

Proposition 4.7. The boundary at infinity $\partial_{\infty} \mathcal{G}$ of a tile graph $\mathcal{G}(f, \mathcal{C})$ can be identified with $\mathbb{S}^{2}$. Under this identification, a metric $d$ is a visual metric on $\mathbb{S}^{2}$ with respect to the expanding Thurston map $f$ if and only if $d$ is a visual metric on $\partial_{\infty} \mathcal{G}$ (in the sense of Gromov hyperbolic spaces).

Here the metric $d$ on $\partial_{\infty} \mathcal{G}$ means the pull-pack metric of $d$ under the identification.

Proof. Let $d$ be a visual metric with expansion factor $\Lambda$ of $\mathbb{S}^{2}$ with respect to $f$.

For any sequence $\left\{X_{n}\right\}$ converging to $\infty$

$$
\lim _{i, j \rightarrow \infty}\left(X_{i}, X_{j}\right)=\infty
$$

we have a filtration

$$
\bigcup_{i=1}^{\infty} X_{i} \supset \bigcup_{i=2}^{\infty} X_{i} \supset \ldots \supset \bigcup_{i=n}^{\infty} X_{i} \supset \bigcup_{i=n+1}^{\infty} X_{i} \supset \ldots
$$

with

$$
\operatorname{diam}\left(\bigcup_{i=n}^{\infty} X_{i}\right) \rightarrow 0 \text { as } n \rightarrow \infty .
$$

Hence, there exists a limit point $x \in \mathbb{S}^{2}$ such that for any $\epsilon>0$, there exists $N>0$ such that for all $n>N$,

$$
\bigcup_{i=n}^{\infty} X_{i} \subset N_{\epsilon}(x),
$$

where $N_{\epsilon}(x)$ is an $\epsilon$-neighborhood of $x$ in $\mathbb{S}^{2}$, i.e.,

$$
X_{n} \subset N_{\epsilon}(x),
$$

or

$$
d\left(x, X_{n}\right)<\epsilon .
$$

We claim that the limit point is unique. Indeed, if there exists $y \in \mathbb{S}^{2}$ also satisfying (16), then

$$
d(x, y) \leq d\left(x, \operatorname{diam}\left(X_{n}\right)\right)+d\left(y, \operatorname{diam}\left(X_{n}\right)\right) \rightarrow 0 \text { as } n \rightarrow \infty .
$$

Hence, $x=y$. Let $\left\{Y_{n}\right\}$ be an sequence converging to infinity equivalent to $\left\{X_{i}\right\}$, i.e.,

$$
\lim _{i \rightarrow \infty}\left(X_{i}, Y_{i}\right)=\infty
$$


We claim that the limit point of $\left\{Y_{n}\right\}$ is $x$. Indeed, by Lemma 4.2, we have

$$
d\left(x, Y_{n}\right) \leq d\left(x, X_{n}\right)+d\left(Y_{n}, X_{n}\right) \leq d\left(x, X_{n}\right)+k \Lambda^{-\left(Y_{n}, X_{n}\right)} \rightarrow 0
$$

as $n$ goes to infinity since $\left(Y_{n}, X_{n}\right) \rightarrow \infty$. Hence, any two equivalent sequences converging to infinity have the same limit point, and we can assign a limit point to an equivalence class of sequences converging to infinity.

We define

$$
h: \partial_{\infty} \mathcal{G} \rightarrow \mathbb{S}^{2}
$$

by mapping any equivalence class of sequences converging to infinity to its limit point. For any $x \in \mathbb{S}^{2}$, there exists $X_{i}$ with $\ell\left(X_{i}\right)=i$ containing $x$, for any $i \geq-1$. Then by Lemma 4.2, we have that

$$
\begin{aligned}
\left(X_{i}, X_{j}\right) & \geq-\log _{\Lambda} \operatorname{diam}\left(X_{i} \cup X_{j}\right)+\log k \\
& \geq-\log _{\Lambda}\left(\min \left\{\operatorname{diam}\left(X_{i}\right), \operatorname{diam}\left(X_{j}\right)\right\}\right)+\log k \rightarrow \infty
\end{aligned}
$$

as $i, j \rightarrow \infty$, where $k \geq 1$ is a constant as in Lemma 4.2. So $\left\{X_{i}\right\}$ is a converging sequence with limit point $x$. Hence, the map $h$ is surjective. In order to prove the injectivity, for any two sequences converging to infinity $\left\{X_{i}\right\}$ and $\left\{Y_{i}\right\}$, we let $x$ and $y$ be their limit points respectively. If $x=y$, then

$$
\operatorname{diam}\left(X_{n} \cup Y_{n}\right) \rightarrow 0 \text { as } n \rightarrow \infty .
$$

So by Lemma 4.1 and 4.3 .

$$
\left(X_{n}, Y_{n}\right) \geq m\left(X_{n}, Y_{n}\right)-1 \geq-\log _{\Lambda}\left(C \operatorname{diam}\left(X_{n} \cup Y_{n}\right)\right)-1 \rightarrow \infty
$$

as $n$ goes to infinity, which implies that $\left\{X_{i}\right\}$ and $\left\{Y_{i}\right\}$ are equivalent. Hence, $h$ is injective.

We only need to show that that there exists a constant $C>0$ such that for any $\xi, \xi^{\prime} \in \partial_{\infty} \mathcal{G}, x=h(\xi)$ and $y=h\left(\xi^{\prime}\right)$,

$$
\frac{1}{C} \Lambda^{-\left(\xi, \xi^{\prime}\right)} \leq d(x, y) \leq C \Lambda^{-\left(\xi, \xi^{\prime}\right)} .
$$

Pick any $\left\{X_{n}\right\} \in \xi$ and $\left\{Y_{n}\right\} \in \xi^{\prime}$. By Lemma 4.1

$$
\frac{1}{C} \Lambda^{-\left(X_{n}, Y_{n}\right)} \leq \operatorname{diam}\left(X_{n} \cup Y_{n}\right) \leq C \Lambda^{-\left(X_{n}, Y_{n}\right)} .
$$

Taking the limit superior, we get

$$
\frac{1}{C} \limsup _{n \rightarrow \infty} \Lambda^{-\left(X_{n}, Y_{n}\right)} \leq \lim _{n \rightarrow \infty} \operatorname{diam}\left(X_{n} \cup Y_{n}\right) \leq C \limsup _{n \rightarrow \infty} \Lambda^{-\left(X_{n}, Y_{n}\right)} .
$$

Hence, we have

$$
\begin{aligned}
\frac{1}{C} \Lambda^{-\liminf _{n \rightarrow \infty}\left(X_{n}, Y_{n}\right)} \leq \lim _{n \rightarrow \infty} \operatorname{diam}( & \left.X_{n} \cup Y_{n}\right) \\
& \leq C \Lambda^{-\liminf _{n \rightarrow \infty}\left(X_{n}, Y_{n}\right)}
\end{aligned}
$$

Since

$$
d(x, y)=\lim _{n \rightarrow \infty} \operatorname{diam}\left(X_{n}, Y_{n}\right)
$$


and

$$
\left(\xi, \xi^{\prime}\right) \leq \liminf _{n \rightarrow \infty}\left(X_{n}, Y_{n}\right)
$$

by inequality (17)

$$
\begin{aligned}
d(x, y) & =\lim _{n \rightarrow \infty} \operatorname{diam}\left(X_{n} \cup Y_{n}\right) \\
& \leq C \Lambda^{-\liminf _{n \rightarrow \infty}\left(X_{n}, Y_{n}\right)} \\
& \leq C \Lambda^{-\left(\xi, \xi^{\prime}\right)} .
\end{aligned}
$$

Since

$$
\left(\xi, \xi^{\prime}\right)=\inf \liminf _{n \rightarrow \infty}\left(X_{n}, Y_{n}\right)
$$

where infimum is taken for all $\left\{X_{n}\right\} \in \xi$ and $\left\{Y_{n}\right\} \in \xi^{\prime}$, by inequality (17),

$$
\frac{1}{C} \sup \Lambda^{-\liminf _{n \rightarrow \infty}\left(X_{n}, Y_{n}\right)} \leq \lim _{n \rightarrow \infty} \operatorname{diam}\left(X_{n} \cup Y_{n}\right)=d(x, y)
$$

where supremum is taken for all $\left\{X_{n}\right\} \in \xi$ and $\left\{Y_{n}\right\} \in \xi^{\prime}$. Hence,

$$
\frac{1}{C} \Lambda^{-\left(\xi, \xi^{\prime}\right)}=\frac{1}{C} \Lambda^{-\inf \liminf _{n \rightarrow \infty}\left(X_{n}, Y_{n}\right)} \leq d(x, y) .
$$

Combining equations (18) and (19), we get that

$$
\frac{1}{C} \Lambda^{-\left(\xi, \xi^{\prime}\right)} \leq d(x, y) \leq 4 C \Lambda^{-\left(\xi, \xi^{\prime}\right)},
$$

SO

$$
\frac{1}{C} \Lambda^{-\left(\xi, \xi^{\prime}\right)} \leq d\left(h(\xi), h\left(\xi^{\prime}\right)\right) \leq C \Lambda^{-\left(\xi, \xi^{\prime}\right)}
$$

for all $\xi, \xi^{\prime} \in \partial_{\infty} \mathcal{G}$. Therefore, the pull-back of the metric $d$ on $\mathbb{S}^{2}$ under $h$ is a visual metric on $\partial_{\infty} \mathcal{G}$.

Since $d$ is a visual metric with respect to $f$, equation (20) implies that there exists a constant $c \geq 0$ such that for all $x, y \in \mathbb{S}^{2}$, and $\xi=h^{-1}(x), \xi=h^{-1}(y)$,

$$
\left(\xi, \xi^{\prime}\right)-c \leq m(x, y) \leq\left(\xi, \xi^{\prime}\right)+c .
$$

Let $\rho$ be a visual metric on $\partial_{\infty} \mathcal{G}$ on the Gromov hyperbolic space, so there exists constant $k \geq 1$, such that for any $\xi, \xi^{\prime} \in \partial_{\infty} \mathcal{G}$,

$$
\frac{1}{k} \Lambda^{-\left(\xi, \xi^{\prime}\right)} \leq \rho\left(\xi, \xi^{\prime}\right) \leq k \Lambda^{-\left(\xi, \xi^{\prime}\right)} .
$$

By equation (21), there exists a constant $k^{\prime} \geq 1$, such that

$$
\frac{1}{k^{\prime}} \Lambda^{-(x, y)} \leq \frac{1}{k} \Lambda^{-\left(\xi, \xi^{\prime}\right)} \leq \rho\left(h^{-1}(x), h^{-1}(y)\right) \leq k \Lambda^{-\left(\xi, \xi^{\prime}\right)} \leq k^{\prime} \Lambda^{-m(x, y)},
$$

where $x, y \in \mathbb{S}^{2}, \xi=h^{-1}(x)$ and $\xi=h^{-1}(y)$. Therefore, the pull-back of the metric $\rho$ on $\partial_{\infty} \mathcal{G}$ under $h^{-1}$ is a visual metric on $\mathbb{S}^{2}$. 
For any Jordan curves $\mathcal{C}$ and $\mathcal{C}^{\prime}$ containing post $(f)$, let $\partial_{\infty} \mathcal{G}=$ $\partial_{\infty} \mathcal{G}(f, \mathcal{C})$ and $\partial_{\infty} \mathcal{G}^{\prime}=\partial_{\infty} \mathcal{G}\left(f, \mathcal{C}^{\prime}\right)$ be the boundary at infinity of the tile graphs $\mathcal{G}(f, \mathcal{C})$ and $\mathcal{G}\left(f, \mathcal{C}^{\prime}\right)$ respectively. By the proposition above, there exist identifications

$$
h: \partial_{\infty} \mathcal{G} \rightarrow \mathbb{S}^{2}
$$

and

$$
h^{\prime}: \partial_{\infty} \mathcal{G}^{\prime} \rightarrow \mathbb{S}^{2}
$$

So we have the following diagram

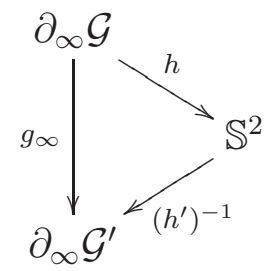

This induced bijection $g_{\infty}=\left(h^{\prime}\right)^{-1} \circ h$ should be the same as $g_{\infty}$ as in the remark after Proposition 4.6. In addition, under this identification, visual metrics on $\partial_{\infty} \mathcal{G}$ and $\partial_{\infty} \mathcal{G}^{\prime}$ are also identified. This is the following corollary.

Corollary 4.8. For any Jordan curves $\mathcal{C}$ and $\mathcal{C}^{\prime}$ containing post $(f)$, there exists a natural identification between $\mathcal{G}=\partial_{\infty} \mathcal{G}(f, \mathcal{C})$ and $\mathcal{G}^{\prime}=$ $\partial_{\infty} \mathcal{G}\left(f, \mathcal{C}^{\prime}\right)$. Under this identification, a metric $\rho$ is a visual metric on $\partial_{\infty} \mathcal{G}$ if and only if it is a visual metric on $\partial_{\infty} \mathcal{G}^{\prime}$.

\section{Asymptotic Upper Curvature}

In this section, we define the asymptotic upper curvature for an expanding Thurston map. After review the definition of Lattès maps, we give a curvature characterization of Lattès maps.

Let $f: \mathbb{S}^{2} \rightarrow S^{2}$ be an expanding Thurston map. We define the asymptotic upper curvature of $f$ as

$$
K_{u}(f):=K_{u}(\mathcal{G}(f, \mathcal{C})),
$$

where $\mathcal{C} \subset \mathbb{S}^{2}$ is any Jordan curve containing post $(f)$ and $\mathcal{G}=\mathcal{G}(f, \mathcal{C})$ denotes the Gromov hyperbolic graph constructed from the cell decompositions of $(f, \mathcal{C})$. For any Jordan curves $\mathcal{C}$ and $\mathcal{C}^{\prime}$, the Gromov hyperbolic space $\mathcal{G}(f, \mathcal{C})$ and $\mathcal{G}\left(f, \mathcal{C}^{\prime}\right)$ are rough-isomeric by Proposition 4.6, and the asymptotic upper curvature is invariant under rough-isometry, SO

$$
K_{u}(\mathcal{G}(f, \mathcal{C}))=K_{u}\left(\mathcal{G}\left(f, \mathcal{C}^{\prime}\right)\right) .
$$

Therefore, the asymptotic upper curvature $K(f)$ is well-defined in equation (22). 
A Lattès map $f: \widehat{\mathbb{C}} \rightarrow \widehat{\mathbb{C}}$ is a rational map that is obtained from a finite quotient of a conformal torus endomorphism, i.e., the map $f$ satisfies the following commutative diagram:

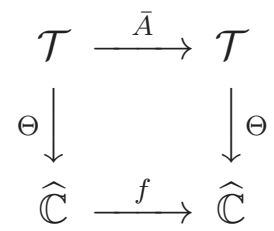

where $\bar{A}$ is a map of a torus $\mathcal{T}$ that is a quotient of an affine map of the complex plane, and $\Theta$ is a finite-to-one holomorphic map. Lattès maps were the first examples of rational maps whose Julia set is the whole sphere $\widehat{\mathbb{C}}$, and a Lattès map is an expanding Thurston map. In [Y], we have the following combinatorial characterization of Lattès maps:

Theorem 5.1 (Yin, 2011). A map $f: \mathbb{S}^{2} \rightarrow \mathbb{S}^{2}$ is topologically conjugate to a Lattès map if and only if the following conditions hold:

- $f$ is an expanding Thurston map;

- $f$ has no periodic critical points;

- there exists $c>0$ such that $D_{n} \geq c(\operatorname{deg} f)^{n / 2}$ for all $n>0$.

We have the following statement(see Corollary 8.2 in [Y]).

Corollary 5.2. A map $f: \mathbb{S}^{2} \rightarrow \mathbb{S}^{2}$ is topologically conjugate to a Lattès map if and only if the followings conditions hold:

- $f$ is an expanding Thurston map;

- $f$ has no periodic critical points;

- there exists a visual metric on $\mathbb{S}^{2}$ with respect to $f$ with expansion factor $\Lambda=\operatorname{deg}(f)^{1 / 2}$.

This leads to an curvature characterization of Lattès maps as follows.

Theorem 5.3. Let $f: \mathbb{S}^{2} \rightarrow \mathbb{S}^{2}$ be an expanding Thurston map. The asymptotic upper curvature of $f$ satisfies

$$
K_{u}(f) \geq-\frac{1}{4} \log ^{2}(\operatorname{deg} f) .
$$

If in addition, the map $f$ has no periodic critical points, then the tile graph $\mathcal{G}=\mathcal{G}(f)$ is an $A C_{u}(\kappa)$-space with

$$
\kappa=-\frac{1}{4} \log ^{2}(\operatorname{deg} f),
$$

if and only if the map $f$ is topologically conjugate to a Lattès map.

Proof. The first part follows directly from the definition of asymptotic upper curvature of $f$ and from Theorem 3.2 and Theorem 2.13.

If $f$ is topologically conjugate to a Lattès map, then by Corollary 5.2, there exists a visual metric on $\mathbb{S}^{2}$ with respect to $f$ with expansion factor $\Lambda=\operatorname{deg}(f)^{1 / 2}$. By Proposition 4.7, there exists a visual metric on $\partial_{\infty} \mathcal{G}$ in the sense of Gromov hyperbolic spaces with expansion factor 
$\Lambda=\operatorname{deg}(f)^{1 / 2}$. By Theorem 3.2, the Gromov hyperbolic space $\mathcal{G}$ is an $\mathrm{AC}_{u}(\kappa)$-space with

$$
\kappa=-\frac{1}{4} \log ^{2}(\operatorname{deg} f) .
$$

Conversely, if $\mathcal{G}$ is an $\mathrm{AC}_{u}(\kappa)$-space with

$$
\kappa=-\frac{1}{4} \log ^{2}(\operatorname{deg} f),
$$

then for all $X, X^{\prime} \in \mathcal{G}$ and all finite sequences $X_{0}=X, X_{1}, \ldots, X_{n}=X^{\prime}$ in $\mathcal{G}$,

$$
\left(X, X^{\prime}\right) \geq \min _{i=1,2, \ldots, n}\left(X_{i-1}, X_{i}\right)-\frac{\log n}{\log (\operatorname{deg} f)^{1 / 2}}-c .
$$

Let $D_{n}$ be the minimum number of $n$-tiles needed to join opposite sides of Jordan curve $\mathcal{C}$ as defined in (7), for $n>0$. For $\#$ post $(f) \geq 4$, let $P_{n}=X_{1} \ldots X_{D_{n}}$ be an $n$-tile chain joining opposite sides of $\mathcal{C}$. By the equation (24), we have

$$
\left(X_{1}, X_{D_{n}}\right) \geq \min _{i=1,2, \ldots, D_{n}}\left(X_{i-1}, X_{i}\right)-\frac{\log D_{n}}{\log (\operatorname{deg} f)^{1 / 2}}-c,
$$

SO

$$
\frac{\log D_{n}}{\log (\operatorname{deg} f)^{1 / 2}} \geq \min _{i=1,2, \ldots, D_{n}}\left(X_{i-1}, X_{i}\right)-\left(X_{1}, X_{D_{n}}\right)-c .
$$

By equation (13), we have

$$
\begin{aligned}
\left(X_{i-1}, X_{i}\right) & =\frac{1}{2}\left[\ell\left(X_{i-1}\right)+\ell\left(X_{i}\right)-\eta\left(X_{i-1}, X_{i}\right)\right]+1 \\
& =\frac{1}{2}\left[2 n-\eta\left(X_{i-1}, X_{i}\right)\right]+1 \\
& \geq n+\frac{1}{2}
\end{aligned}
$$

where $\eta\left(X_{i-1}, X_{i}\right)=1$ since $\eta$ is the path metric, and $X_{i-1}$ and $X_{i}$ have nonempty intersection. Applying equation (26) and Lemma 4.2 to equation (25), we have

$$
\begin{aligned}
\frac{\log D_{n}}{\log (\operatorname{deg} f)^{1 / 2}} & \geq \min _{i=1,2, \ldots, D_{n}}\left(X_{i-1}, X_{i}\right)-\left(X_{1}, X_{D_{n}}\right)-c \\
& \geq n+\frac{1}{2}+\log _{\Lambda}\left(\operatorname{diam}\left(X_{1} \cup X_{D_{n}}\right)\right)-\log _{\Lambda} k-c,
\end{aligned}
$$

where $k \geq 1$ only depends on $f$ and $\mathcal{C}$ as in Lemma 4.2, and $N>0$ only depends on $f$. Let $d$ be the minimum length of a line segment joining opposite sides of the Jordan curve, then $d>0$ and

$$
\operatorname{diam}\left(X_{1} \cup X_{D_{n}}\right) \geq d .
$$


So

$$
\begin{aligned}
\frac{\log D_{n}}{\log (\operatorname{deg} f)^{1 / 2}} & \geq n+\frac{1}{2}+\log _{\Lambda}(d)-\log _{\Lambda} k-c \\
& =n+C .
\end{aligned}
$$

Here the constant

$$
C=\frac{1}{2}+\log _{\Lambda}(d)-\log _{\Lambda} k-c
$$

only depends on $f, \mathcal{C}$ and $\Lambda$. Hence, we have

$$
\log D_{n} \geq(n+C) \log (\operatorname{deg} f)^{1 / 2} .
$$

Therefore, we have

$$
D_{n} \geq C^{\prime}(\operatorname{deg} f)^{n / 2}
$$

where $C^{\prime}=(\operatorname{deg} f)^{C / 2}$ only depends on $f$ and $\mathcal{C}$.

When \# post $(f)=3$, the argument above holds with minor modifications. More specifically, consider a set $S$ of $D_{n} n$-tiles joining opposite sides of $\mathcal{C}$. By a simple compactness argument, there exists a positive lower bound $d$ on the diameter of any set intersecting all three edges of $\mathcal{C}$. Hence, we may choose $n$-tiles $X$ and $Y$ in $S$ that intersect two distinct 0 -edges and satisfy $\operatorname{diam}(X \cup Y) \geq d$. Then one can choose $P_{n}=X_{1} \ldots X_{r_{n}}$ to be an $n$-tile chain joining opposite sides of $\mathcal{C}$, such that $X_{1}=X, X_{r_{n}}=Y, X_{i} \in S$ for $i=1, \ldots, r_{n}$, and $r_{n}<2 D_{n}$.

By Theorem [5.1, the map $f$ is topologically conjugate to a Lattès map. 


\section{REFERENCES}

[A] Lars Ahlfors, Remarks on Kleinian groups, Tulane Symp. on Quasiconformal Mappings (1967).

[BF] Mario Bonk and Thomas Foertsch, Asymptotic upper curvature bounds in coarse geometry, Math. Z. 253 (2006), 753-785.

[BM] Mario Bonk and Daniel Meyer, Expanding Thurston maps, Preprint (2010). arXiv:1009.3647v1.

[BP] Marc Bourdon and Hervé Pajot, Cohomologie $l_{p}$ et espaces de Besov, J. Reine Angew. Math. 558 (2003), 85-108.

[BS] Sergei Buyalo and Viktor Schroeder, Elements of asymptotic geometry, EMS Monographs in Mathematics, European Mathematical Society, Zürich, 2007.

[CRSR] C. Correia Ramos and J. Sousa Ramos, Kleinian groups and holomorphic dynamics, Internat. J. Bifur. Chaos Appl. Sci. Engrg. 13 (2003), no. 7, 1959-1967. Dynamical systems and functional equations (Murcia, 2000).

[DH] Adrien Douady and John H. Hubbard, A proof of Thurston's topological characterization of rational functions, Acta Math. 171 (1993), 263-297.

$[\mathrm{H}]$ Ursula Hamenstädt, Entropy-rigidity of locally symmetric spaces of negative curvature, Ann. of Math. (2) 131 (1990), 35-51.

[HP] Peter Haïssinsky and Kevin M. Pilgrim, Coarse expanding conformal dynamics, Astérisque 325 (2009).

[MSS] R. Mañé, P. Sad, and D. Sullivan, On the dynamics of rational maps, Ann. Sci. École Norm. Sup. (4) 16 (1983), no. 2, 193-217.

[NS] Hossein Namazi and Juan Souto, Non-realizability and ending laminations: Proof of the density conjecture, Acta Math. 209 (2012), no. 2, 323-395.

[O] Ken'ichi Ohshika, Realising end invariants by limits of minimally parabolic, geometrically finite groups, Geom. Topol. 15 (2011), no. 2, 827-890.

$[\mathrm{P}]$ Kevin M. Pilgrim, Julia sets as Gromov boundaries following V. Nekrashevych, Topology Proc. 29 (2005), 293-316.

[S] Dennis Sullivan, Quasiconformal homeomorphisms and dynamics. I. Solution of the Fatou-Julia problem on wandering domains, Ann. of Math. (2) 122 (1985), 401-418.

[Y] Qian Yin, Lattès maps and combinatorial expansion, Preprint, 2010.

Qian Yin, The University of Chicago, 5734 S. University Avenue, Chicago, Illinois 60637, USA

E-mail address: qyin@math.uchicago.edu 